\title{
Recommendations for Therapeutic Apheresis by the Section "Preparative and Therapeutic Hemapheresis" of the German Society for Transfusion Medicine and Immunohematology
}

\author{
Nina Worel ${ }^{a} \quad$ Behrouz Mansouri Taleghani $^{b}$ Erwin Strasser ${ }^{c}$ \\ ${ }^{a}$ Department for Blood Group Serology and Transfusion Medicine, Medical University Vienna, Vienna, Austria; \\ b University Clinic of Hematology and Central Hematology Laboratory, Division of Transfusion Medicine,

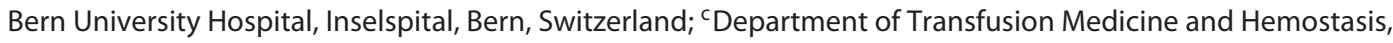 \\ University Hospital Erlangen, Erlangen, Germany
}

\section{Keywords}

Therapeutic apheresis · Plasma exchange · Leukodepletion · Extracorporeal photopheresis

\begin{abstract}
The section "Preparative and Therapeutic Hemapheresis" of the German Society for Transfusion Medicine and Immunohematology (DGTI) has reviewed the actual literature and updated techniques and indications for evidence-based use of therapeutic apheresis in human disease. The recommendations are mostly in line with the "Guidelines on the Use of Therapeutic Apheresis in Clinical Practice" published by the Writing Committee of the American Society for Apheresis (ASFA) and have been conducted by experts from the DACH (Germany, Austria, Switzerland) region.
\end{abstract}

(c) 2019 S. Karger AG, Base

\section{Introduction}

The section "Preparative and Therapeutic Hemapheresis" of the German Society for Transfusion Medicine and Immunohematology (DGTI) presents recommendations for therapeutic apheresis (TA). Experts from Austria, Germany, and Switzerland reviewed the actual literature including the most recent guidelines of the American Society for Apheresis (ASFA) [1].
TA requires a thorough diagnostic work-up, a close co-operation of involved medical specialties, and an experienced apheresis team. In TA, whole blood is transiently removed from the circulation and components contributing to the disease are removed after separation. In case of plasma treatments, eliminated plasma may be replaced. TA is based on two assumptions: (1) the disease is caused by pathological or pathologically elevated or missing blood constituents; (2) TA is sufficiently effective in removing the causative constituents or in replacing missing factors in order to achieve a symptomatic remission [2].

TA procedures classified as cytaphereses include red blood cell (RBC) depletion or exchange, leukocyte depletion (LD), platelet depletion, and extracorporeal photopheresis (ECP). Plasma treatments include therapeutic plasma exchange (TPE; exchange of plasma using a replacement fluid) and various secondary treatments of separated plasma comprising adsorption, filtration, or precipitation of selective or specific components with or without the need of replacement fluids. Whole blood adsorption methods are characterized by the direct removal of cellular and non-cellular blood components from whole blood (i.e., without a primary cell or plasma separation) with or without fluid replacement.

\section{KARGER}

(c) 2019 S. Karger AG, Basel 


\section{General Considerations}

\section{Organization of Centers}

Apheresis units need to provide adequate conditions as reported for preparative apheresis [3]. The medical director of an apheresis facility is normally board-certified in an appropriate medical specialty (e.g., transfusion medicine, internal medicine, pediatrics). Physicians and operators (nurses, technicians) need to be experienced in extracorporeal procedures and educated in emergency medicine. Specific training for each apheresis device is required. Physicians performing TA should possess hands-on experience with at least 10 apheresis treatments per apheresis system and continuously update their education. An operator should not perform more than two TA procedures in parallel. All technical equipment including apheresis devices and secondary separation systems (selective/specific removal procedures) should be approved for the respective application, CE certified, and handled according to national legislation [3-6].

\section{Patient Information and Consent}

The attending physician is responsible for informing the patient and/or the legal guardian verbally in a competent and understandable manner about the value and necessity of the therapeutic measure, the risks and benefits and potential side effects, as well as alternative treatment options. We suggest that the patient receives a copy of the informed consent, providing sufficient time for consideration for decision making and is advised to immediately report any occurring symptoms during TA.

\section{Apheresis Procedures}

The indication, treatment schedule, and therapeutic goal needs to be assessed in close co-operation with physicians involved in patient care. Appropriate fluid replacement should be considered when removing large volumes during TA. The efficacy of a procedure can be calculated based on the composition of the blood components to be removed, the kinetic of reappearance, renewed synthesis, or redistribution of disease-associated factors. Intensity and frequency of treatments are based on clinical conditions, treatment goals, and laboratory results.

The operator has to check and document the device and tubing set, use of correct fluids (e.g., anti-coagulant, replacement fluids) based on the patient requirements and therapy-relevant data. For TA, peripheral venous access (i.e., antecubital veins) using dialysis cannulas and/ or peripheral venous catheters, is preferred. Currently, ultrasound devices are more and more frequently used to facilitate peripheral vein puncture. All patients should be adequately monitored at a minimum blood pressure, heart rate, and oxygen saturation. Additional monitoring may be applied if necessary, such as ECG, etc. A physician has to be available during the whole TA procedure in case of medical or technical problems. TA needs to be stopped if the clinical condition of the patient deteriorates, or in case of any severe adverse events (AEs) or system malfunction. The responsible physician has to decide whether to continue, interrupt, or discontinue the procedure, and is responsible for the discharge/transfer of the patient after apheresis.

Patients should receive adequate fluid replacement with proper documentation of fluid balance. A warming system for patients and/or for the extracorporeal blood and replacement solutions is advisable to avoid side effects of the procedure. This is also a requirement in all patients with cold agglutinin disease or cryoglobulinemia.

Especially in plasma treatments, elimination of drugs (e.g., antibiotics, etc.) should be taken into account and preferably applied after the TA. Systematic studies about the effect of plasma differential separation methods on drug efficacy do not exist. Thus, drugs should be taken after the apheresis treatment if feasible.

Lipemic or highly icteric plasma may interfere with the optical sensors of the apheresis device. Therefore, parenteral nutrition should be stopped if possible $4-6 \mathrm{~h}$ before the start of the apheresis procedure.

\section{Laboratory Tests}

Laboratory test results (i.e., complete peripheral blood count, calcium, potassium, and coagulation parameters) should be available before the first TA treatment. Specific laboratory tests for monitoring the response to TA (i.e., ADAMTs-13 and LDH in TTP, etc.), should be assessed accordingly.

\section{Anticoagulation}

Centrifugal apheresis devices use citrate anticoagulation (acid-citrate-dextrose formula A, ACD-A) in a ratio of usually 1:10 to 1:22 ACD-A to whole blood, depending on the type of apheresis, clinical or laboratory parameters, or citrate re-transfusion (i.e., plasma adsorption treatments) to the patients. The effect of citrate is based on the chelation of calcium. Citrate-related side effects are frequent and can be lowered by reducing the flow rates and adjusting the $\mathrm{ACD}-\mathrm{A}$ ratio. In addition, calcium gluconate $10 \%$ may be administered on demand or as continuous infusion $\left(0.53-1.2 \mathrm{mmol} \mathrm{Ca}{ }^{2+}\right.$ per $10 \mathrm{mmol}$ citrate) [7-10]. For bolus administration in adults, $10 \mathrm{~mL}$ of calcium gluconate $10 \%(2.1 \mathrm{mmol})$ should be given over a period of at least $10 \mathrm{~min}$. Continuous calcium substitution is strongly recommended in patients with a concentration of ionized calcium plasma $<0.75 \mathrm{mmol} / \mathrm{L}$ [8]. The threshold for $\mathrm{Ca}^{2+}$ substitution in the event of a severe citrate reaction is $0.9 \mathrm{mmol} / \mathrm{L}$. The infusion rate of 
citrate should not exceed the upper limit of $1.2 \mathrm{~mL} / \mathrm{min}$ ACD-A per liter blood volume without prophylactic calcium supplementation. It is recommended that pediatric patients receive calcium gluconate at a lower, age-adapted dose.

Citrate is mainly metabolized in the liver and to a lesser extent in the kidneys and skeletal muscle. Patients with liver disease show a trend to increased citrate toxicity while patients with kidney dysfunction are at increased risk of metabolic alkalosis, especially during TPE with plasma as the replacement fluid $[11,12]$. Alkalosis reduces citrate metabolism and may aggravate side effects from hypocalcemia [2]. Hyperventilation may in addition intensify symptoms of alkalosis.

To avoid citrate accumulation, heparin can be added after contraindication (e.g., bleeding or bleeding risk) have been ruled out. If heparin is used, monitoring of coagulation is indicated by assessing the activated partial thromboplastin time or activated coagulation time before starting and after the procedure [13]. In addition, patients should be monitored for bleeding symptoms.

Heparin-induced thrombocytopenia type II is an absolute contraindication for the use of heparin. In these very rare cases, heparin needs to be replaced by alternative anticoagulants such as argatroban, a direct thrombin inhibitor. However, there are only few reports about argatroban and extracorporeal therapy in the context of dialysis and extracorporeal membrane oxygenation [14].

\section{AEs and Complications}

AEs can occur suddenly or delayed. Especially in children, because of limited communication a careful monitoring (surveillance, vital signs, laboratory tests) is a required imperative. AE during apheresis typically consist of citrate toxicity which can be increased in patients on bisphosphonates therapy, vasovagal reactions and hypotension, hypothermia, and allergic reactions, for example, and can result from concomitant medication (e.g., angiotensin-converting enzyme [ACE] inhibitors). According to the WAA registry study, additional complications from peripheral or central venipunctures, coagulation disorders, or hypo- and hypervolemia (impaired fluid balance) can occur. Mechanical hemolysis and iron deficits (due to remaining blood in the tubing set) are rare complications. The possibility of air embolism, especially when using a central venous line (CVC), is extremely rare [15].

Paresthesia - perioral or peripheral - are among the most common citrate-induced side effects. However, severe citrate reactions such as tetania, hypernatremia, or metabolic alkalosis/acidosis are rare. Patients with a central venous line are at risk for cardiac arrhythmia if high blood flow rates are applied as a high citrate concentration can affect the conduction system of the heart $[2,16]$.
The first symptoms of vasovagal reactions are pallor and cold sweats, followed by bradycardia and arterial hypotension. More severe reactions include nausea, vomiting, syncope, and rarely seizures with or without enuresis. In the case of a vasovagal reaction, the patient has to be brought into shock position until he or she recovers and the apheresis procedure should be transiently stopped.

Complications associated with peripheral vein puncture include hematoma, arterial puncture, extravasation injury, thrombophlebitis, and vascular wall sclerosis after multiple apheresis procedures. Nerve injuries with consecutive peripheral sensory or motor deficits or accidental arterial puncture occur more rarely.

Allergic reactions are usually triggered by replacement fluids containing protein, but also by other colloidal solutions, residual ethylene oxide after sterilization in the tubing set or filters, or by concomitant medication. The symptoms range from mild erythema, hives, tachycardia, hypotension, bronchospasm, and allergic shock. Adequate medication has to be applied (e.g., antihistamines, steroids, or epinephrine and volume). Depending on the severity, the procedure has to be interrupted or terminated.

Special precautions should be taken in patients treated with ACE inhibitors. The contact of blood with negatively charged surfaces (e.g., LDL apheresis using dextran sulfate cellulose or cascade filtration) can activate the kininkallikrein system. ACE inhibitors block the decay of bradykinin and at the same time inhibit the production of the vaso-constricting angiotensin II, which functions as a potential bradykinin antagonist. Therefore, the use of ACE inhibitors is contraindicated in patients undergoing apheresis and we strongly suggest that the treatment is scheduled in accordance with the half-life of the respective ACE inhibitor [17].

\section{Apheresis in Pediatric Patients}

Apheresis in children requires special considerations, including rooms with adequate space to accommodate an accompanying person and a comfortable atmosphere for children. Notably, teams carrying out apheresis in pediatric patients are required to have specific experience, particularly if the body weight (BW) is below $20 \mathrm{~kg}$. Therefore, a team can be considered experienced after carrying out at least five pediatric apheresis procedures in addition to an educational program for TA as reported above for adults. Designated team members have to be trained in emergency medicine and cardiopulmonary resuscitation in children.

As a general rule, priming with cross-matched, preheated packed RBCs, optionally irradiated, hematocrit adjusted using compatible plasma or human albumin is recommended when the $\mathrm{BW}$ is less than $20 \mathrm{~kg}$ and the extracorporeal volume exceeds the total blood volume 
(TBV) by $10-15 \%$ [18]. AEs in children may present differently than in adults: unusual behavior, restlessness, or even sudden silence can be a first symptom that requires a re-evaluation of the situation. Especially in children, a system of warming should be used to avoid hypothermia. As the number of therapeutic aphereses in children is low, large, randomized trials are lacking and indications for TA are adopted from recommendations for adults. It has to be considered that apheresis devices are generally developed for their use in adults and specific systems for children are not available. Important considerations are the disproportion between extra- and intracorporal blood volume, difficult venous access, low flow rates, and other aspects concerning the physiology as well as psychology of pediatric patients. Because of the lack of experience in pediatric apheresis procedures, it is strongly recommended to perform each treatment in co-operation with pediatricians and pediatric caregivers who should be available on demand during apheresis [19]. The monitoring of children during apheresis depends on their condition and should at least include continuous monitoring of heart rate, oxygen saturation, and blood pressure in regular intervals. Necessarily, personnel need to be trained on the specific monitoring equipment for children and its use. Sedation or anesthesia of children during apheresis is usually not compulsory but may be evaluated in individual cases by the attending physician. To avoid citrate toxicity, prophylactic calcium supplementation is recommended when apheresis in children is performed. Instrument settings need to be discussed with the manufacturer due to specific recommendations for pediatric aphereses [20].

Preferably, a peripheral venous line should be used. Broviac or Hickman catheters are appropriate in very small children if the catheter lumen provides sufficient blood flow rates. Apheresis in patients with less than $4 \mathrm{~kg}$ BW who have approximately $240 \mathrm{~mL}$ TBV would lead to a blood exchange as the extracorporeal volume of most apheresis devices is $160-200 \mathrm{~mL}$.

Patients aged below 14 years need be informed about the planned procedure together with their legal guardian in an age-appropriate manner, while patients of 14 years or older have to be directly included in the decision-making process and have to consent considering the national law.

\section{Documentation}

Referral of a patient for TA should be done in written form. Treatment plan, therapeutic endpoint (i.e., target values), as well as follow-up visits have to be specified in consultation with the referring physician. The handling of blood products, management of transfusion reactions, responsibilities, and documentation has to be in accordance with national law and European regulations.
Documentation of each TA, including details of the procedure, lot numbers of the used disposables, type and quantity of solutions, timeline, vital parameters, laboratory test results, and if appropriate additional information, deviations, and AEs, is required. Written reports addressing details of the procedure and the clinical course of the patient are a relevant information for the referring physician.

Termination of apheresis, AEs, procedures to prevent AEs, interventions, and treatments need to be accurately recorded, particularly in the case of transfusion reactions. Investigating and reporting of AEs has to be done by the responsible physician. Reporting of AEs (e.g., transfusion reactions, severe AEs regarding the procedure) and technical problems (e.g., malfunction of the instrument, tubing set) have to be reported to the regulatory agency and if appropriate to the manufacturer according to the national requirements.

\section{TA Procedures}

Treatment modalities, categories, and grade of recommendation according to the ASFA guidelines are summarized in Table 1 [1].

\section{Category Definitions for TA}

I. Disorders for which apheresis is accepted as the firstline therapy, either as a primary standalone treatment or in conjunction with other modes of treatment.

II. Disorders for which apheresis is accepted as second-line therapy, either as a standalone treatment or in conjunction with other modes of treatment.

III. Optimum role of apheresis therapy is not established. Decision making should be individualized.

IV. Disorders in which published evidence demonstrates or suggests apheresis to be ineffective or harmful. IRB approval is desirable if apheresis treatment is undertaken in these circumstances.

\section{Grading Recommendations}

1A Strong recommendation, high-quality evidence.

1B Strong recommendation, moderate-quality evidence.

1C Strong recommendation, low-quality or very low-quality evidence.

2A Weak recommendation, high-quality evidence.

2B Weak recommendation, moderate-quality evidence.

2C Weak recommendation, low-quality or very lowquality evidence. 
Table 1. Indications, category, and grade recommendations for TA

\begin{tabular}{|c|c|c|c|c|c|}
\hline $\begin{array}{l}\text { Specialty, indication, diagnosis - } \\
\text { ASFA guidelines } 2019\end{array}$ & Modality & $\begin{array}{l}\text { Category/ } \\
\text { grade }\end{array}$ & $\begin{array}{l}\text { Specialty, indication, diagnosis - } \\
\text { ASFA guidelines } 2019\end{array}$ & Modality & $\begin{array}{l}\text { Category/ } \\
\text { grade }\end{array}$ \\
\hline \multicolumn{3}{|l|}{ Hematology } & \multicolumn{3}{|l|}{ Thrombotic microangiopathy, infection associated } \\
\hline AB0-major incompatible HCT (apheresis) & TPE & $\mathrm{II} / 2 \mathrm{~B}$ & STEC-HUS, severe & TPE/IA & $\mathrm{III} / 2 \mathrm{C}$ \\
\hline AB0-major incompatible HCT (bone marrow) & TPE & $\mathrm{II} / 1 \mathrm{~B}$ & pHUS & TPE & $\mathrm{III} / 2 \mathrm{C}$ \\
\hline $\mathrm{AB} 0$-minor or bidirectional incompatible HCT & RBC-E & $\mathrm{III} / 2 \mathrm{C}$ & Thrombotic microangiopathy, transplant associated & TPE & $\mathrm{III} / 2 \mathrm{C}$ \\
\hline \multicolumn{3}{|c|}{ Red cell alloimmunization in pregnancy (non-RhD) } & \multicolumn{3}{|l|}{ Thrombocytosis } \\
\hline (prior to intra-uterine transfusion availability) & TPE & $\mathrm{III} / 2 \mathrm{C}$ & Symptomatic & TA & $\mathrm{II} / 2 \mathrm{C}$ \\
\hline \multirow{2}{*}{\multicolumn{3}{|c|}{$\begin{array}{l}\text { Prevention of RhD alloimmunization after RBC } \\
\text { exposure, exposure to } \mathrm{RhD}(+) \mathrm{RBCs}\end{array}$}} & Prophylactic or secondary & TA & $\mathrm{III} / 2 \mathrm{C}$ \\
\hline & $\begin{array}{l}\mathrm{RBC}-\mathrm{E} \\
\mathrm{TPE}\end{array}$ & $\mathrm{III} / 2 \mathrm{C}$ & \multicolumn{3}{|l|}{ Metabolic Diseases } \\
\hline Aplastic anemia & TPE & $\mathrm{III} / 2 \mathrm{C}$ & \multirow{3}{*}{$\begin{array}{l}\text { Acute liver failure/therapy refractory pruritus } \\
\text { HV TPE }\end{array}$} & & \\
\hline Pure red cell aplasia & $\mathrm{TPE}$ & $\mathrm{III} / 2 \mathrm{C}$ & & TPE & $111 / 2 \mathrm{~B}$ \\
\hline AIHA, warm AIHA & TPE & $\mathrm{III} / 2 \mathrm{C}$ & & TPE-HV & $\mathrm{I} / 1 \mathrm{~A}$ \\
\hline Cold agglutinin disease & TPE & $\mathrm{III} / 2 \mathrm{C}$ & \multicolumn{2}{|l|}{ Familial hypercholesterolemia } & \\
\hline Severe cold agglutinin disease & TPE & $\mathrm{II} / 2 \mathrm{C}$ & Homozygotes & LDL aph & $\mathrm{I} / 1 \mathrm{~A}$ \\
\hline \multirow{2}{*}{ Amyloidosis, systemic } & B2 MG & $\mathrm{II} / 2 \mathrm{~B}$ & Heterozygotes & LDL aph & II/1A \\
\hline & TPE & $\mathrm{IV} / 2 \mathrm{C}$ & Homozygotes/heterozygotes & TPA & $\mathrm{II} / 1 \mathrm{~B}$ \\
\hline Babesiosis & RBC-E & $\mathrm{II} / 2 \mathrm{C}$ & \\
\hline \multicolumn{3}{|l|}{ GVHD } & Severe HTG-AP & TPE/ & $\mathrm{III} / 1 \mathrm{C}$ \\
\hline $\begin{array}{l}\text { Acute } \\
\text { Chronic }\end{array}$ & $\begin{array}{l}\text { ECP } \\
\text { FCP }\end{array}$ & $11 / 1 \mathrm{C}$ & & LDL aph & $\mathrm{III} / 2 \mathrm{C}$ \\
\hline $\begin{array}{l}\text { Chronic } \\
\text { Hereditary hemochromatosis }\end{array}$ & $\begin{array}{l}\text { ECP } \\
\text { EA }\end{array}$ & $11 / 1 \mathrm{~B}$ & Prevention of severe HTG-AP & TPE/ & \\
\hline \multirow[t]{2}{*}{ Coagulation factor inhibitors } & TPE & $\mathrm{III} / 2 \mathrm{C}$ & & LDL aph & \\
\hline & IA & $\mathrm{III} / 2 \mathrm{~B}$ & \multicolumn{3}{|l|}{ Overdose, envenomation, and poisoning } \\
\hline \multicolumn{3}{|l|}{ Hyperleukocytosis } & Mushroom poisoning & $\mathrm{TPE}$ & $\mathrm{II} / 2 \mathrm{C}$ \\
\hline Symptomatic & LD & $\mathrm{II} / 2 \mathrm{~B}$ & Envenomation & TPE & $\mathrm{III} / 2 \mathrm{C}$ \\
\hline Prophylactic or secondary & LD & $\mathrm{III} / 2 \mathrm{C}$ & Drug overdose/poisoning & TPE & $\mathrm{III} / 2 \mathrm{C}$ \\
\hline \multicolumn{3}{|l|}{ Hyperviscosity in monoclonal gammopathies } & Neurology & & \\
\hline Symptomatic & TPE & $\mathrm{I} / 1 \mathrm{~B}$ & Acute disseminated encephalomyelitis, steroid & & \\
\hline $\begin{array}{l}\text { Prophylaxis for rituximab } \\
\text { Immune thrombertonenia }\end{array}$ & $\mathrm{TPE}$ & $\mathrm{I} / 1 \mathrm{C}$ & $\begin{array}{l}\text { Acute disseminated encephalomyelitis, steroid } \\
\text { refractory }\end{array}$ & TPE & $\mathrm{II} / 2 \mathrm{C}$ \\
\hline $\begin{array}{l}\text { Immune thrombocytopenia } \\
\text { Refractory }\end{array}$ & TPE/IA & $\mathrm{III} / 2 \mathrm{C}$ & Acute inflammatory demyelinating & $\mathrm{TPE}$ & $\mathrm{I} / 1 \mathrm{~A}$ \\
\hline Cryoglobulinemia, severe/symptomatic & TPA & $\mathrm{II} / 2 \mathrm{~A}$ & polyradiculoneuropathy (Guillain-Barre syndrome), & & \\
\hline & IA & $\mathrm{II} / 2 \mathrm{~B}$ & primary treatment & IA & $\mathrm{I} / 1 \mathrm{~B}$ \\
\hline Cutaneous T cell lymphoma; mycosis fungoides; & & & Chronic focal encephalitis (Rasmussen encephalitis) & $\mathrm{TPE}$ & $\mathrm{III} / 2 \mathrm{C}$ \\
\hline Sezary syndrome & & & Chronic inflammatory demyelinating & & \\
\hline Erythrodermic mycosis fungoides III, Sezary & & & polyradiculoneuropathy & TPE/IA & $\mathrm{I} / 1 \mathrm{~B}$ \\
\hline syndrome & ECP & $\mathrm{I} / 1 \mathrm{~B}$ & Lambert-Eaton myasthenic syndrome & TPE & $\mathrm{II} / 2 \mathrm{C}$ \\
\hline Non-erythrodermic & ECP & $\mathrm{III} / 2 \mathrm{C}$ & Multiple sclerosis & & \\
\hline Malaria, severe & RBC-E & $\mathrm{III} / 2 \mathrm{~B}$ & Acute attack/relapse & TPE & $\mathrm{II} / 1 \mathrm{~A}$ \\
\hline Polycythemia vera & EA & $\mathrm{I} / 1 \mathrm{~B}$ & Acute attack/relapse & IA & $\mathrm{II} / 1 \mathrm{~B}$ \\
\hline Secondary erythrocytosis & EA & $\mathrm{III} / 1 \mathrm{C}$ & Chronic & TPE/IA & $\mathrm{III} / 2 \mathrm{~B}$ \\
\hline Post transfusion purpura & TPE & $\mathrm{III} / 2 \mathrm{C}$ & Neuromyelitis optica spectrum disorders & & \\
\hline Sickle cell disease, acute & & & Acute attack/relapse & TPE & $\mathrm{II} / 1 \mathrm{~B}$ \\
\hline Acute stroke & RBC-E & $\mathrm{I} / 1 \mathrm{C}$ & Acute attack/relapse & IA & $\mathrm{II} / 1 \mathrm{C}$ \\
\hline Acute chest syndrome, severe & RBC-E & $\mathrm{II} / 1 \mathrm{C}$ & Maintenance & TPE & $\mathrm{III} / 2 \mathrm{C}$ \\
\hline Other complications & RBC-E & $\mathrm{III} / 2 \mathrm{C}$ & Myasthenia gravis & & \\
\hline Sickle cell disease, non-acute & & & Moderate-severe & TPE & $\mathrm{I} / 1 \mathrm{~B}$ \\
\hline Stroke prophylaxis/iron overload prevention & RBC-E & $\mathrm{I} / 1 \mathrm{~A}$ & Pre-thymectomy & $\mathrm{TPE}$ & $\mathrm{I} / 1 \mathrm{C}$ \\
\hline Recurrent vaso-occlusive pain crisis & RBC-E & $\mathrm{II} / 2 \mathrm{~B}$ & N-methyl-D-aspartate receptor antibody encephalitis & TPE & $\mathrm{I} / 1 \mathrm{C}$ \\
\hline Pre-operative management & RBC-E & $\mathrm{III} / 2 \mathrm{~A}$ & PANDAS associated with streptococcal infections & & \\
\hline Pregnancy & RBC-E & $\mathrm{II} / 2 \mathrm{~B}$ & PANDAS exacerbation & $\mathrm{TPE}$ & $\mathrm{II} / 1 \mathrm{~B}$ \\
\hline Thrombotic thrombocytopenic purpura & TPE & $\mathrm{I} / 1 \mathrm{~A}$ & Sydenham's chorea, severe & TPE & $\mathrm{III} / 2 \mathrm{~B}$ \\
\hline Thrombotic microangiopathy, coagulation mediated & & & Paraneoplastic neurological syndromes & TPE/IA & $\mathrm{III} / 2 \mathrm{C}$ \\
\hline THBD, DGKE, and PLG mutations & TPE & $\mathrm{III} / 2 \mathrm{C}$ & Paraproteinemic demyelinating neuropathies & & \\
\hline Thrombotic microangiopathy, complement & & & Anti-myelin-associated glycoprotein neuropathy & $\mathrm{TPE}$ & $\mathrm{III} / 1 \mathrm{C}$ \\
\hline mediated & & & Multifocal motor neuropathy & TPE & $\mathrm{IV} / 1 \mathrm{C}$ \\
\hline Factor $\mathrm{H}$ autoantibodies & TPE & $\mathrm{I} / 2 \mathrm{C}$ & IgG/IgA/IgM & $\mathrm{TPE}$ & $\mathrm{I} / 1 \mathrm{~B}$ \\
\hline Complement factor gene mutations & TPE & $\mathrm{III} / 2 \mathrm{C}$ & Multiple myeloma & TPE & $\mathrm{III} / 2 \mathrm{C}$ \\
\hline Thrombotic microangiopathy, drug associated & & & Progressive multifocal leukoencephalopathy & & \\
\hline HCT & TPE & $\mathrm{III} / 2 \mathrm{C}$ & associated with natalizumab & $\mathrm{TPE}$ & $\mathrm{I} / 1 \mathrm{C}$ \\
\hline Ticlopidine & TPE & $\mathrm{I} / 2 \mathrm{~B}$ & Stiff-person syndrome & TPE & $\mathrm{III} / 2 \mathrm{C}$ \\
\hline Clopidogrel & $\mathrm{TPE}$ & $\mathrm{III} / 2 \mathrm{~B}$ & & & \\
\hline Gemcitabine/quinine & TPE & $\mathrm{IV} / 2 \mathrm{C}$ & & & \\
\hline
\end{tabular}




\begin{tabular}{|c|c|c|c|c|c|}
\hline $\begin{array}{l}\text { Specialty, indication, diagnosis - } \\
\text { ASFA guidelines } 2019\end{array}$ & Modality & $\begin{array}{l}\text { Category/ } \\
\text { grade }\end{array}$ & $\begin{array}{l}\text { Specialty, indication, diagnosis - } \\
\text { ASFA guidelines } 2019\end{array}$ & Modality & $\begin{array}{l}\text { Category/ } \\
\text { grade }\end{array}$ \\
\hline \multicolumn{3}{|l|}{ Nephrology } & HELLP syndrome & & \\
\hline \multirow{2}{*}{\multicolumn{3}{|c|}{$\begin{array}{l}\text { ANCA-associated vasculitis (granulomatosis with } \\
\text { polyangiitis; microscopic polyangiitis) }\end{array}$}} & Postpartum & TPE & $\mathrm{III} / 2 \mathrm{C}$ \\
\hline & & & Antepartum & TPE & $\mathrm{IV} / 2 \mathrm{C}$ \\
\hline Dialysis dependence & TPE & $\mathrm{I} / 1 \mathrm{~A}$ & Hemophagocytic lymphohistiocytosis; & & \\
\hline $\begin{array}{l}\text { Dialysis dependence with diffuse alveolar } \\
\text { hemorrhage }\end{array}$ & TPE & $\mathrm{I} / 1 \mathrm{C}$ & $\begin{array}{l}\text { hemophagocytic syndrome; macrophage-activating } \\
\text { syndrome }\end{array}$ & TPE & $\mathrm{U \amalg} / 2 \mathrm{C}$ \\
\hline Dialysis independence & TPE & $\mathrm{III} / 2 \mathrm{C}$ & Sudden sensorineural hearing loss & LDL aph. & $\mathrm{III} / 2 \mathrm{~A}$ \\
\hline \multirow{2}{*}{\multicolumn{3}{|c|}{$\begin{array}{l}\text { Anti-glomerular basement membrane disease } \\
\text { (Goodpasture's syndrome) }\end{array}$}} & & $\mathrm{RH} / \mathrm{TPE}$ & $\mathrm{III} / 2 \mathrm{~A}$ \\
\hline & & & Heparin-induced thrombocytopenia and thrombosis & & \\
\hline Dialysis dependence, no diffuse alveolar & & & Pre-cardiopulmonary bypass & TPE & $\mathrm{III} / 2 \mathrm{C}$ \\
\hline hemorrhage & TPE & $\mathrm{III} / 2 \mathrm{~B}$ & Thrombosis & TPE & $\mathrm{III} / 2 \mathrm{C}$ \\
\hline Dialysis dependence, diffuse alveolar & & & Voltage-gated potassium channel antibodies & TPE/IA & $\mathrm{II} / 1 \mathrm{~B}$ \\
\hline hemorrhage & TPE & $\mathrm{I} / 1 \mathrm{C}$ & Age-related macular degeneration, dry & RH & $\mathrm{II} / 2 \mathrm{~B}$ \\
\hline Dialysis independence & TPE & $\mathrm{I} / 1 \mathrm{~B}$ & Wilson's disease, fulminant & TPE & $\mathrm{I} / 1 \mathrm{C}$ \\
\hline Catastrophic antiphospholipid syndrome & TPE & $\mathrm{I} / 2 \mathrm{C}$ & Phytanic acid storage disease (Refsum's disease) & TPE/ & $\mathrm{II} / 2 \mathrm{C}$ \\
\hline & & LDL aph & \\
\hline Recurrent in kidney transplant & TPE & $\mathrm{I} / 1 \mathrm{~B}$ & Sepsis with multi-organ failure & $\mathrm{TPE}$ & $\mathrm{III} / 2 \mathrm{~B}$ \\
\hline \multicolumn{3}{|c|}{ Recurrent in kidney transplant/steroid resistant } & Thyroid storm & TPE & $\mathrm{II} / 2 \mathrm{C}$ \\
\hline in native kidney & LDL aph. & $\mathrm{II} / 2 \mathrm{C}$ & Nephrogenic systemic fibrosis & $\mathrm{ECP/TPE}$ & $\mathrm{III} / 2 \mathrm{C}$ \\
\hline Steroid resistant in native kidney & TPE & $\mathrm{III} / 2 \mathrm{C}$ & Pemphigus vulgaris, severe & $\mathrm{TPE}$ & $\mathrm{III} / 2 \mathrm{~B}$ \\
\hline \multicolumn{3}{|l|}{ Immunoglobulin A nephropathy } & & $\mathrm{ECP} / \mathrm{IA}$ & $\mathrm{III} / 2 \mathrm{C}$ \\
\hline Crescentic & TPE & $\mathrm{III} / 2 \mathrm{~B}$ & Psoriasis, disseminated pustular & ECP & $\mathrm{III} / 2 \mathrm{~B}$ \\
\hline Chronic progressive & TPE & $\mathrm{III} / 2 \mathrm{C}$ & & $\mathrm{ADC}$ & $\mathrm{III} / 2 \mathrm{C}$ \\
\hline Myeloma cast nephropathy & TPE & $\mathrm{II} / 2 \mathrm{~B}$ & & TPE & $\mathrm{IV} / 2 \mathrm{C}$ \\
\hline \multirow[t]{2}{*}{ Scleroderma (systemic sclerosis) } & TPE & $\mathrm{III} / 2 \mathrm{C}$ & Toxic epidermal necrolysis, refractory & TPE & $\mathrm{III} / 2 \mathrm{~B}$ \\
\hline & ECP & $\mathrm{III} / 2 \mathrm{~A}$ & Vasculitis IgA Henoch-Schönlein purpura & & \\
\hline & TPE & $11 / 2 \mathrm{C}$ & Crescentic & TPE & $\mathrm{III} / 2 \mathrm{C}$ \\
\hline \multicolumn{3}{|l|}{ Solid organ transplantation } & Severe extrarenal disease & TPE & $\mathrm{III} / 2 \mathrm{C}$ \\
\hline \multicolumn{3}{|l|}{ Cardiac transplantation } & Vasculitis & & \\
\hline Cellular/recurrent rejection & ECP & $\mathrm{II} / 1 \mathrm{~B}$ & HBV-associated polyarteriitis nodosa (PAN) & TPE & $\mathrm{II} / 2 \mathrm{C}$ \\
\hline Rejection prophylaxis & ECP & $\mathrm{II} / 2 \mathrm{~A}$ & Idiopathic PAN & TPE & $\mathrm{IV} / 1 \mathrm{~B}$ \\
\hline Desensitization & TPE & $\mathrm{II} / 1 \mathrm{C}$ & Behcet's disease & $\mathrm{ADC}$ & $\mathrm{II} / 1 \mathrm{C}$ \\
\hline Antibody mediated rejection & TPE & $\mathrm{III} / 2 \mathrm{C}$ & Behcet's disease & TPE & $\mathrm{III} / 2 \mathrm{C}$ \\
\hline Liver transplantation $\mathrm{AB} 0$ incompatible & & & Burn shock resuscitation & TPE & $\mathrm{III} / 2 \mathrm{~B}$ \\
\hline
\end{tabular}

Desensitization, living donor $\quad$ TPE I/1C

Desensitization, deceased donor $\quad$ TPE III/2C

Antibody-mediated rejection (AB0 and HLA) TPE III/2C

Desensitization, ABO incompatible $\quad$ ECP III/2C

Antibody rejection/immune suppression $\quad$ ECP III/2B

Lung transplantation

Bronchiolitis obliterans syndrome $\quad$ ECP II/1C

Antibody-mediated rejection/desensitization TPE III/2C

Renal transplantation, AB0 compatible

Antibody mediated rejection $\quad$ TPE/IA I/1B

Desensitization, living donor $\quad$ TPE/IA I/1B

Desensitization, deceased donor (high panelreactivity)

Renal transplantation, $\mathrm{AB} 0$ incompatible

Desensitization, living donor

Antibody-medicated rejection

TPE/IA III/2C

TPE/IA I/1B

TPE/IA II/1B

Various diseases

Atopic (neuro-)dermatitis (atopic eczema),

recalcitrant

Inflammatory bowel disease

Ulcerative colitis/Crohn's disease $\quad$ ADC III/1B

Crohn's disease $\quad$ ECP III/2C

Complex regional pain syndrome, chronic $\quad$ TPE $\quad$ III/2C

Dilated cardiomyopathy, idiopathic, NYHA II-IV IA II/1B

TPE III/2C

Erythropoietic porphyria, liver disease

$\mathrm{EA} / \mathrm{TPE} \quad \mathrm{III} / 2 \mathrm{C}$

Hashimoto's encephalopathy: steroid-responsive

encephalopathy associated with autoimmune

thyroiditis

TPE $\quad$ II $/ 2 \mathrm{C}$
Adapted from Padmanabhan et al. [1]. TA, therapeutic apheresis; ADC adsorptive cytapheresis; AIHA, autoimmune hemolytic anemia; GVHD, graft versus host disease; PV, polycythemia vera; HCT, hematopoietic stem cell transplantation; $\mathrm{Lp}(\mathrm{a})$, lipoprotein a; ANCA, anti-neutrophil cytoplasmatic antibodies; ASFA, American Society for Apheresis, c., column; DGKE, diacylglycerol kinase epsilon; EA, erythrocytapheresis; ECP, extracorporeal photopheresis; HCT, hematopoietic cell transplantation; HELLP, hemolysis, elevated liver enzymes, and low platelets; HTG-AP, hypertriglyceridemic pancreatitis; HV, high volume; IA, immunoadsorption; LA, lymphapheresis; LD, leukodepletion; LDL aph., LDL apheresis; NYHA, New York Heart Association; PANDAS, pediatric autoimmune neuropsychiatric disorders; pHUS, thrombotic microangiopathy due to Streptococcus pneumoniae; PLG, plasminogen; RBCs, red blood cells; RBC-E, red blood cell exchange; RhD, Rhesus D; RH, rheohemapheresis; STEC-HUS, Shiga toxin-mediated thrombotic microangiopathy; THBD, thrombomodulin; TPE, therapeutic plasma exchange.

\section{Therapeutic Plasma Exchange}

TPE is defined as the non-specific and non-selective removal of plasma and its replacement with an iso-oncotic solution. TPE is an option in disorders where known or unknown pathogens need to be removed or a missing plasma component has to be replaced [1]. TPE represents the longest practiced method of TA until now and has the most category I indications defined in the ASFA guidelines [1]. 
After separation of whole blood by centrifugation or membrane filtration, plasma is removed while blood cells are returned together with the replacement fluid.

Selection of the replacement fluid and its volume depend on the underlying disease, concomitant disorders, specific laboratory parameters (e.g., total serum protein, coagulation factors) and the patients' fluid balance to maintain isovolumic conditions and a normal oncotic pressure. It normally consists of colloid solutions (e.g., albumin and/or plasma) or a combination of crystalloid and colloid solution [21].

The exchange volume is normally $1.0-1.5$ times the patient's total plasma volume with therapy intervals of 24-48 $\mathrm{h}$ depending on the underlying diseases, clinical feasibility, kinetics of the pathological plasma components, and its distribution and half-life. In the case of autoantibodies, their binding avidity to the target tissue, renewed synthesis, and extravascular to intravascular redistribution of the respective substance has also to be taken into account. Drugs with high protein binding $(>80 \%)$ and low volume of distribution $(<0.2 \mathrm{~L} / \mathrm{kg})$ are efficiently eliminated by TPE [22]. Therefore, if possible, patients should receive their daily medication (especially if the application is once a day) after TPE, and women should be aware that oral contraceptives may be less effective. TPE is in general a low-risk procedure. However, $\mathrm{AE}$ as citrate toxicity or hypersensitivity to plasma or human albumin has to be considered [23].

\section{Selective Plasma Separation}

After the primary step of plasma separation, substances of interest can be removed either by filtration, adsorption (immunological or electrostatic/physicochemical), or precipitation followed by continuous re-infusion of the treated plasma. Replacement fluids are usually not required as the anticoagulation fluid is completely re-infused, thus citrate toxicity may be more frequent than in TPE.

Plasma differential separation is applied when TPE is not sufficient to effectively remove pathogens. Severe forms of autosomal dominant homozygotic familial hypercholesterolemia are ASFA category I indications. Drug intolerance and individual atherosclerosis risks have to be taken into consideration as well $[1,24]$.

Membrane differential filtration (MDF) is often referred to as cascade filtration or rheohemapheresis. Agerelated dry macular degeneration is an ASFA category I indication for rheohemapheresis treatment [25]. Primary separation of the whole blood is followed by plasma filtration. MDF uses filters with defined surface properties and pore size to selectively separate plasma proteins based on their molecular weight and conformation for elimination of LDL cholesterol and its fraction lipoprotein a $[\operatorname{Lp}(a)]$, as well as $\alpha_{2}$-macroglobulin, IgM, triglycerides, fibrinogen, and coagulation factor XIII.

\section{Cryofiltration}

Cryofiltration is a special type of MDF. A particular set of filters is used to remove large amounts of cryoproteins from the plasma at low temperature $\left(4^{\circ} \mathrm{C}\right)$ [26].

\section{Immunoadsorption}

Immunoadsorption (IA) is an apheresis procedure aimed to remove immune complexes and auto- and alloantibodies. Adsorption can be achieved by hydrophobic interaction chromatography, specific affinity chromatography, or through immunological binding of pathogens to their ligands (immobilized as amino acids, peptides, or proteins).

At therapy onset, daily IA is feasible. In disorders where antibody titers correlate with disease severity, such as coagulation factor inhibitor hemophilia, reduction of antibody titers can be used to assess the efficacy of IA [27]. Usually, immunosuppressive therapy is combined with IA, which classifies inhibitor hemophilia (autoantibody) as an ASFA category III indication, for example [1]. The choice of adsorption columns, frequency of IA, and plasma volume to be processed depend on the underlying disease, its severity, and the capacity of the adsorber. Due to the specific device the treatment intensity may be limited by simultaneous elimination of plasma proteins with a long half-life (e.g., factor XIII, fibrinogen). Substitution with immunoglobulins can be required or may be part of the treatment protocol.

\section{Lipid Apheresis (LDL Apheresis)}

TPE plays only a minimal role in treating severe lipid metabolism disorders that are based on the chylomicronemia syndrome. For LDL apheresis there are two FDAapproved methods: the heparin-induced extracorporeal LDL precipitation system, and the dextran sulfate LDL removal column. Heparin-induced extracorporeal LDL precipitation (HELP ${ }^{\circledR}$ - LDL apheresis) is the most complex form of lipid apheresis. The basic principle is to precipitate LDL using heparin. In addition to LDL, VLDL, and $L p(a)$, mediators of inflammation, fibrinogen, proinflammatory adhesion molecules, and other cytokines are eliminated [28-30]. Direct adsorption of lipoproteins (DALI) from whole blood is carried out with special (DALI)-adsorbers and thus does not require additional plasma separation steps. LDL and Lp(a) diffuse into the pores of the disposable adsorber, while corpuscular blood components are retained [31].

IA was described for selective removal of apoB100containing lipoproteins and Lp(a) [32]. By use of the latter selective procedure, only one lipid molecule is removed, whereas the HELP system diminishes more and different proteins, such as LDL-A, LDL-C, and fibrinogen.

All methods for LA (IA, chemoadsorption, precipitation, MDF, and adsorption from whole blood) meet the 
criteria of a minimum $60 \%$ reduction of LDL cholesterol within $6 \mathrm{~h}$ of the therapy session. The most specific and effective method is the immuno-specific adsorption of Apo-B-carrying lipoproteins [26]. LDL adsorption with dextran sulfate cellulose can cause severe reactions due to bradykinin release. It is advisable to withhold ACE inhibitors for at least $24 \mathrm{~h}$ before apheresis treatment to avoid severe reactions.

\section{Therapeutic Erythrocytapheresis}

Therapeutic erythrocytapheresis (EA) is performed by an apheresis device, replacing a defined amount of RBCs with cell-free electrolyte solution (RBC depletion). The selective removal of RBCs aims to lower hematocrit levels in polycythemia vera (PV) or iron overload in hereditary hemochromatosis. Increased hematocrit is associated with hyperviscosity, which can result in fatigue, shortness of breath, angina pectoris, or thrombosis. The main problem of iron overload results in organ impairment or organ failure. As EA is more efficient than classical phlebotomy, EA requires fewer treatments, resulting in longer treatment intervals, and should be the preferred technique in suitable patients (e.g., appropriate antecubital veins, acceptable cardiac function) [33, 34]. EA may be carried out up to 2-3 times per week until the target hematocrit is reached [35]. If the blood volume is assumed to be $70 \mathrm{~mL} / \mathrm{kg}$, the following formula is used to estimate the volume of red cells to be removed in the apheresis procedure $(\mathrm{VR}): \mathrm{VR}=[($ starting $\mathrm{HCT}$ - target $\mathrm{HCT}) / 79]$ $\times[70(\mathrm{~mL} / \mathrm{kg}) \times \mathrm{kg} \mathrm{BW}]$ [36]. The risk associated with therapeutic EA is the same as with other apheresis procedures. Special care needs to be taken in patients with organ impairment, particularly cardiac arrhythmias, cardiopulmonary insufficiency, and liver failure, as the risks of AE may be increased.

\section{RBC Exchange}

The principal of RBC exchange (RBC-E) is to replace a patient's RBCs with at least an equal volume of compatible packed red cells. RBC-E is used in patients with hemoglobinopathies $[37,38]$ or diseases caused by an intraerythrocytic protozoan (e.g., malaria, babesiosis) [39, 40]. The advantage of the RBC-E over an exchange transfusion is the controllability, the closed, sterile apheresis system, as well as reduced exposure to donor plasma. RBC-E is well tolerated and, in contrast to classical exchange transfusion, allows the monitoring of fluid balance.

RBC-E in patients with intravascular hemolysis can exacerbate the damage of disease-affected RBCs [41]. Patients with homozygous sickle cell anemia, thalassemia major, or combined congenital hemoglobinopathies require regular RBC transfusion, which can result in iron overload and secondary organ impairment if inadequate iron chelation is applied. With RBC-E the risk of iron overload may be re- duced significantly [42]. Moreover, RBC-E seems to reduce the frequency of alloimmunization and may therefore be considered a reasonable alternative to regular transfusions, but is particularly recommended in patients with sickle cell disease in the case of acute central nervous system (CNS) infarction and acute pulmonary reaction [43]. Large-caliber needles for venipuncture combined with low withdrawal flow are used to avoid sickling and hemolysis during extracorporeal circulation [44]. Patients that receive RBC-E maintenance therapy should preferably receive fresh RBCs ( $\leq 7$ days old). Comprehensive phenotyping and selection of highly compatible blood products is advisable in order to avoid allo-immunization [45].

In patients with malaria or babesiosis, RBC-E is indicated if more than $10 \%$ of RBCS are infected, or in the case of neuropathy [1]. In total, 1-1.5 times the patient's TBV should be processed to remove $50-70 \%$ of infected erythrocytes. Repeated apheresis in patients with severe disease may achieve a residual parasitemia of less than 5\% [39].

\section{AB0-Incompatible Blood Stem Cell Transplantation}

Due to the fact that the blood group system is inherited independently from the human leukocyte antigen system, approximately $50 \%$ of allogeneic HCT (hematopoietic blood stem cell transplantations) are performed across the blood group barrier. Conditioning regimens prior to HCT can be myeloablative (MA) or non-MA (NMA) [46]. To reduce the overall toxicity of HCT, NMA conditioning is the preferred type of treatment in patients who are older ( $>55$ years) or have comorbidities and are therefore not eligible for an MA-HCT. In NMA, graftversus-host disease (GVHD) prophylaxis does not include methotrexate. As a result, antibody-producing donor B cells can escape T cell control and produce isohemagglutinins, which can lead to hyperacute hemolysis of recipient RBCs in the case of a minor or bidirectional $\mathrm{AB} 0$ incompatibility between donor and recipient [46]. This passenger lymphocyte syndrome can also be observed after AB0 mismatched solid organ transplantation [46]. To avoid this critical complication, a prophylactic RBC-E with donor group RBCs should be considered, provided that a corresponding transfusion regimen was not implemented prior to transplantation $[46,47]$.

\section{Leukocyte Depletion}

LD is performed in patients with acute and chronic leukemia to treat hyperleukocytosis-induced symptoms of leukostasis. Leukostasis is a known risk factor for increased early death in newly diagnosed acute leukemia $[48,49]$. The mechanism of leukostasis remains undefined. However, it is assumed that elevated blood viscosity, diminished deformability, and increased adhesiveness of leukemic cells play a key role [48, 49]. Leukostasis typically affects the lung and the CNS, and results in dys- 
pnea, hypoxia, alveolar hemorrhage, pulmonary infiltrates [22], and neurological symptoms such as impaired consciousness, impaired vision, retinal hemorrhage, and coma. In addition, cardiac symptoms and arterial embolism can occur. Priapism is observed mostly in CML with very high leukocyte counts of $>500,000$ per $\mu \mathrm{L}[48,49]$.

The number of white blood cells (WBCs) that triggers apheresis depends on the type of leukemia. In acute myeloid leukemia [18], myelo-monocytic, monoblastic, and monocytic subtypes especially carry a high risk for leukostasis as AML blasts are large cells with a higher rigidity compared to lymphoblastic cells. In the case of WBCs of $>50,000-100,000$ per $\mu \mathrm{L}$, LD should be initiated if clinical symptoms are present. In contrast, acute or chronic lymphoblastic leukemia may cause leukostasis only if WBCs exceed 400,000 per $\mu \mathrm{L}$.

The WBC count provides some guidance, but the indication for therapeutic or prophylactic LD is mostly based on clinical considerations. There is no strong evidence for prophylactic LD, as only a few cases have been reported. Patients undergoing LD may have severe anemia, thrombocytopenia, and coagulopathy. Apheresis settings and optional replacement fluids depend on the type of leukemia, the number of cells (volume) eliminated during LA, and the corresponding hematological parameters. As processing of 1-time TBV leads only to a reduction of approximately $15-46 \%$ of WBCs, TBV should be processed 2 times to reduce WBCs by $50-86 \%$ [48, 49]. An increase of the centrifuge speed may be necessary for a more effective sedimentation of leukemic blasts. In addition, in some cases with difficult separation (e.g., in chronic myeloid leukemia), hydroxyethyl starch may be added continuously during LD to augment $\mathrm{RBC}$ sedimentation. If the volume of removed cells exceeds $20 \%$ of the TBV, replacement with crystalloids or human albumin is recommended. To avoid a further increase in blood viscosity, it is strongly recommended to transfuse RBCs only after the $\mathrm{LD}$ and in consultation with the referring hematologist, even at very low hemoglobin levels. LD should be repeated until the leukostasis symptoms disappear. LD efficiency may seem lower than expected because leukemic cells can be mobilized during apheresis. The target for prophylactic LD is to reduce the WBC count to $<50,000-100,000$ per $\mu \mathrm{L}$ in $\mathrm{AML}$ and $<400,000$ per $\mu \mathrm{L}$ in acute lymphoblastic leukemia to avoid tumor lyses syndrome if chemotherapy is applied [48]. Fragmentation of leukocytes can lead to falsely elevated potassium concentrations and platelet counts in automated blood cell analyzers [50]. Chemotherapy is vitally important for the prognosis and should not be delayed by LD. A long-term or palliative application of LD can be considered for pregnant women and patients with absolute contraindications for chemotherapy, respectively. Despite patients with leukostasis often being critically ill, there are no reports of serious AEs in the context of LD. The procedure is generally well tolerated if carried out properly [51, 52].

\section{Adsorptive Cytapheresis}

Adsorptive cytapheresis (adsorption-desorptionapheresis; ADA) is a special type of leukocyte apheresis, with the purpose to selectively adsorb activated granulocytes, monocytes, and macrophages. The therapy is carried out either with a special column containing approximately 35,000 cellulose acetate beads or with particular filters consisting of polyester fibers. With the filter approach, especially lymphocytes and platelets are removed, which also have a pro-inflammatory function. Activated leukocytes are selectively removed through Fc receptormediated interaction with immunoglobulins or via complement. This leads to a reduction of activated WBCs in the peripheral blood, which is compensated through the release of immature cells (e.g., CD10-negative granulocytes) from the bone marrow. In addition, ADA treatment is associated with the release of anti-inflammatory interleukin (IL)-1 receptor antagonist, suppression of pro-inflammatory cytokines like TNF- $\alpha$, IL-1 $\beta$, IL- 6 , IL8 , down-modulation of L-selectin, and the chemokine receptor CXCR3, and therefore has an immunomodulatory effect. The removal of peripheral dendritic precursor cells seems also to be of relevance $[53,54]$.

During one treatment session, between $1,800 \mathrm{~mL}$ (column approach) or $3,000 \mathrm{~mL}$ (filter approach) of blood is processed. A treatment cycle usually consists of 5 treatments carried out once a week and can be repeated if necessary. In severe cases or in delayed response, treatment can be intensified. By using the column device, treatment intervals can be intensified to twice a week in the first 2 weeks.

ADA is applied in disorders associated with leukocyte activation such as inflammatory bowel disease (i.e., ulcerative colitis, Crohn's disease) [54]. Recent studies have shown that the column system is more efficient when combined with immunomodulatory treatments (e.g., azathioprine) [55]. It is a complementary alternative in patients not responding to standard therapy, or for those being steroid intolerant. Adsorptive cytapheresis is considered to be a low-risk procedure. In rare cases, headache, fatigue, dizziness, general malaise, or nausea may occur [56].

Other possible applications are autoimmune disorders including rheumatoid arthritis and, on rare occasions, psoriasis or pyoderma gangrenosum [1]. There are currently investigational indications of ADA for Behcet's disease [57]. Hence, treatments should be performed only in randomized trials.

\section{Extracorporeal Photopheresis}

ECP is used for the treatment of T cell-mediated disorders and has been shown to be beneficial for patients with cutaneous $\mathrm{T}$ cell lymphoma and lymphomatoid papulosis 
(each CD30+), GVHD, and rejection of solid allografts [57-62]. During ECP, leukocytes (predominantly MNCs) are separated from whole blood, treated with a photosensitizer (8-methoxypsoralen, 8-MOP), then exposed to UVA light and transfused back into the patient. UVA light activates 8-MOP, leads to the crosslinking of DNA strands which results in apoptosis of lymphoid cells, largely natural killer cells and T cells. After reinfusion, apoptotic lymphoid cells are phagocytosed by immature dendritic cells, which subsequently undergo maturation, present antigenic peptides, and modulate immune responses. Increased dendritic cell differentiation, downregulation of autoreactive $B$ cells, alterations in T helper subset populations and lymphocyte homing antigen display, switch from pro-inflammatory to anti-inflammatory cytokine production, and generation of regulatory $\mathrm{T}$ cells have been described. Nonetheless, the mode of action is still not completely understood, although many achievements have been made over the last decades [63-65].

Different methods are available to carry out the procedure. Inline methods (collection of cells, UVA irradiation, and reinfusion is performed in one system), offline systems (collection of cells with a conventional apheresis device, separate UVA illumination system), and MINI ECP (manual MNC preparation from whole blood, separate UVA illumination system) are used for ECP. Heparin is the standard anticoagulant for inline systems and ACD for offline systems, in patients with a low platelet count and/or gut bleeding, heparin should be avoided.

The peripheral blood WBC count should be $\geq 2 \times$ $10^{9} / \mathrm{L}$ at the start of each treatment to allow sufficient collection of cells. Depending on the technique of ECP (inline, offline, or mini ECP) approximately $10-30 \%$ of the circulating MNC are UVA treated and reinfused.

The extracorporeal blood volume should not exceed $15 \%$ of the TBV during ECP. Otherwise, compensatory therapeutic measures have to be taken. Lipemic or icteric plasma hinders leukocyte collection as it interferes with the light sensors of cell separators and requires manual adaption of the interface by the operator. To avoid lipemia, parenteral nutrition should be paused $4-6 \mathrm{~h}$ before the start of the treatment.

Different therapeutic regimens are recommended depending on the underlying disease. Acute GVHD is treated depending on the severity with 2-3 ECP sessions per week for the first $2-3$ weeks, then twice a week on a weekly basis until the disease response and steroids can be tapered [66, 67]. For chronic GVHD, one cycle weekly for the first month (or biweekly if treating only mucocutaneous chronic GVHD), then in biweekly intervals until either a response or for 8-12 weeks, followed by a taper to every 3-4 weeks until maximal response [66]. Therapy intervals can be adapted depending on organ involvement and response. Response to ECP is first evaluated after 6-8 weeks.
For the prevention and treatment of heart transplant rejection, ECP is performed either according to the protocol published by Barr et al. [68] or scheduled weekly ( 2 days per week), and then every $2-8$ weeks until clinical improvement [69]. In bronchiolitis obliterans syndrome after lung transplantation, ECP is applied weekly ( 2 treatments) in the first month, then 2 treatments every other week for month 2-3, and thereafter 2 treatments per month in months 4-6 [70]. The recommendation for other indications is 2 treatments every $2-4$ weeks. Response has to be evaluated on a regular basis. Re-transfusion of treated cells may lead to fever with or without erythroderma. After 8-MOP treatment, patients are temporarily UVA sensitive and therefore must avoid direct sunlight and wear UVA protective glasses.

\section{Thrombocytapheresis}

Therapeutic thrombocytapheresis or platelet depletion encompasses platelet separation, collection, and removal of a highly enriched platelet concentrate from the circulation of patients. It is accepted as an effective therapeutic option for a rapid reduction of excess of platelets. Thrombocytosis can be reactive (iron-deficiency anemia, malignancies, inflammation, etc.), the result of asplenia but also occur in various myeloproliferative neoplasms (MPN) or overlap syndromes (e.g., essential thrombocythemia, PV). Patients are often affected by thromboembolism or bleeding. The indication for thrombocytapheresis is usually confined to this group of patients. The typical symptoms of MPN with thrombocytosis are microcirculatory disturbances including erythromelalgia, thromboembolism, ischemia of the CNS (visual or speech disorders, dizziness, migraine), or cardiac symptoms (coronary ischemia, myocardial infarction). Thrombocytosis is particularly dangerous in patients with PV who also exhibit a hyperviscosity syndrome with excessively increased hematocrit. In these cases, a rapid simultaneous reduction of the hematocrit as well as the thrombocytes is important [71-73].

Apart from the risk of thromboembolic complications, patients with MPN and thrombocytosis often have an increased risk for bleeding complications as thrombocytosis can be associated with an acquired von Willebrand syndrome [74]. The significance of thrombocytapheresis for reducing the risk of thrombo-hemorrhagic incidences is not clear. Although the procedure is not mentioned in the current guidelines of the German Society of Hematology and Oncology according MPN, it can be useful in specific cases where a rapid decrease in platelet count is indicated (e.g., splenectomy with subsequent high platelet counts, severe or life-threatening circulatory dysfunction, acute bleeding due to an acquired von Willebrand syndrome) or in situations where the established pharmacological therapy is contraindicated $[75,76]$. 
Platelet count can be reduced by $30-60 \%$ per apheresis. At least 1.5 times the patient's TBV should be processed. The cell separator should be set at the highest achievable platelet concentration. The goal of apheresis in patients with acute thromboembolism or bleeding should be the normalization of platelet counts. The number of aphereses depends on the symptoms of the patient [72, 77]. An increase in the ACD-A ratio may become necessary. Cell separator settings for the collection of MNCs may be advantageous in individuals with very high platelet counts (i.e., $>2,000,000 / \mu \mathrm{L}$ ). Platelet depletion is associated with a low risk of side effects.

\section{Conclusion}

TA should only be carried out by experienced teams after careful evaluation of the indication and balance of the risks and benefits. Especially in pediatric patients, this is an absolute requirement. Before starting TA, a treatment plan and aim must be defined and the patient's clinical status has to be re-evaluated at regular intervals.

\section{Acknowledgements}

We want to thank Heidrun Andreu-Ulrich, Hans-Gert Heuft, Kristina Hölig, Sixten Körper, Gerda Leitner, Rainer Moog, Andreas Opitz, Peter Reinhart, Silke Rummler, Gabriele Stiegler, Markus Wisneth, and Volker Witt for their substantive contribution in developing the German Recommendations published at the homepage of the "Deutsche Gesellschaft für Transfusionsmedizin und Immunhämatologie e.V. (DGTI).

\section{Disclosure Statement}

The authors have no conflicts of interest to declare.

\section{Funding Sources}

No funding was provided for the preparation of this article.

\section{Author Contributions}

Nina Worel, Behrouz Mansouri Taleghani, and Erwin Strasser wrote the manuscript.

\section{References}

1 Padmanabhan A, Connelly-Smith L, Aqui N, Balogun RA, Klingel R, Meyer E, et al. Guidelines on the Use of Therapeutic Apheresis in Clinical Practice - EvidenceBased Approach from the Writing Committee of the American Society for Apheresis: The Eighth Special Issue. J Clin Apher. 2019 Jun;34(3):171-354.

2 Mansouri Taleghani B. Therapeutische Hämapherese. In: Volker K, editor. Transfusionsmedizin und Immunhämatologie. 4th ed. Basel: Springer; 2010. Available from: https://doi.org/10.1007/978-3-642-127656_33.

3 Matthes G, Moog R, Radtke H, Wiesneth M, Zingsem J. Durchführung präparativer Hämapheresen zur Gewinnung von Blutbestandteilkonzentraten - Empfehlungen zur präparativen Hämapherese der Deutschen Gesellschaft für Transfusionsmedizin und Immunhämatologie (DGTI). Trans Med Hemother. 2007;34:367-74.

4 Richtlinie zur Gewinnung von Blut und Blutbestandteilen und zur Anwendung von Blutprodukten (Richtlinie Hämotherapie). Gesamtnovelle 2017. Available from: https://www.bundesanzeiger.de/ ebanzwww/wexsservlet?page.navid=to bookmark_official\&bookmark_id= aAYgqkUFS8tt0YXUhbo.

5 Gesetz über Medizinprodukte (Medizinproduktegesetz - MPG). Available from: https://www.gesetze-im-internet.de/mpg/ BJNR196300994.html.

6 Verordnung über das Errichten, Betreiben und Anwenden von Medizinprodukten (Medizinprodukte-Betreiberverordnung MPBetreibV). Available from: https://www. gesetze-im-internet.de/mpbetreibv/ BJNR176200998.html.

7 Bolan CD, Cecco SA, Wesley RA, Horne M, Yau YY, Remaley AT, et al. Controlled study of citrate effects and response to i.v. calcium administration during allogeneic peripheral blood progenitor cell donation. Transfusion. 2002 Jul;42(7):935-46.

8 Bolan CD, Yau YY, Cullis HC, Horwitz ME, Mackall CL, Barrett AJ, et al. Pediatric largevolume leukapheresis: a single institution experience with heparin versus citrate-based anticoagulant regimens. Transfusion. 2004 Feb;44(2):229-38.

9 Buchta C, Macher M, Bieglmayer C, Höcker P, Dettke M. Reduction of adverse citrate reactions during autologous large-volume PBPC apheresis by continuous infusion of calcium-gluconate. Transfusion. 2003 Nov; 43(11):1615-21.

10 Witt V, Fischmeister G, Scharner D, Printz D, Pöttschger U, Fritsch G, et al. Collection efficiencies of MNC subpopulations during autologous CD34+ peripheral blood progenitor cell (PBPC) harvests in small children and adolescents. J Clin Apher. 2001;16(4):161-8.

11 Diaz J, Acosta F, Parrilla P, Sansano T, Bento $M$, Cura $S$, et al. Citrate intoxication and blood concentration of ionized calcium in liver transplantation. Transplant Proc. 1994 Dec;26(6):3669-70.

12 Dzik WH, Kirkley SA. Citrate toxicity during massive blood transfusion. Transfus Med Rev. 1988 Jun;2(2):76-94.

13 Meier JK, Wolff D, Pavletic S, Greinix H, Gosau $\mathrm{M}$, Bertz $\mathrm{H}$, et al.; International Consensus Conference on Clinical Practice in cGVHD. Oral chronic graft-versus-host disease: report from the International Consensus Conference on clinical practice in cGVHD. Clin Oral Investig. 2011 Apr;15(2):127-39.

14 Treschan TA, Schaefer MS, Geib J, Bahlmann A, Brezina T, Werner P, et al. Argatroban versus Lepirudin in critically ill patients (ALicia): a randomized controlled trial. Crit Care. 2014 Oct;18(5):588.

15 Mortzell Henriksson M, Newman E, Witt V, Derfler K, Leitner G, Eloot S, et al. Adverse events in apheresis: an update of the WAA registry data. Transfus Apher Sci. 2016;54(1):2-15.

16 Wiesneth M. Gwinnung und Präparation von peripheren Blutstammzellen. In: Kiefel V, editor. Transfusionsmedizin und Immunhämatologie. 4th ed. Berlin: Springer; 2010.

17 Owen HG, Brecher ME. Atypical reactions associated with use of angiotensin-converting enzyme inhibitors and apheresis. Transfusion. 1994 Oct;34(10):891-4.

18 Witt V, Stegmayr B, Ptak J, Wikström B, Berlin G, Axelsson CG, et al. World apheresis registry data from 2003 to 2007, the pediatric and adolescent side of the registry. Transfus Apheresis Sci. 2008 Dec;39(3):255-60.

19 Kim HC. Therapeutic apheresis in pediatric patients. In: McLeod BC, et al., editors. Apheresis principles and practise. 3rd ed. Bethesda: AABB; 2010.

20 Kim HC. Therapeutic pediatric apheresis. J Clin Apher. 2000;15(1-2):129-57.

21 McLeod BC. Plasma and plasma derivatives in therapeutic plasmapheresis. Transfusion. 2012 May;52 Suppl 1:38S-44S.

22 Ibrahim RB, Liu C, Cronin SM, Murphy BC, Cha R, Swerdlow P, et al. Drug removal by plasmapheresis: an evidence-based review. Pharmacotherapy. 2007 Nov;27(11):1529-49. 
23 Williamson LM, Lowe S, Love EM, Cohen H, Soldan K, McClelland DB, et al. Serious hazards of transfusion (SHOT) initiative: analysis of the first two annual reports. BMJ. 1999 Jul; 319(7201):16-9.

24 Thompson GR, Catapano A, Saheb S, AtassiDumont M, Barbir M, Eriksson M, et al. Severe hypercholesterolaemia: therapeutic goals and eligibility criteria for LDL apheresis in Europe. Curr Opin Lipidol. 2010 Dec;21(6): 492-8.

25 Klingel R, Fassbender C, Fassbender T, Göhlen B. Clinical studies to implement Rheopheresis for age-related macular degeneration guided by evidence-based-medicine. Transfus Apheresis Sci. 2003 Aug;29(1):7184.

26 Siami GA, Siami FS. Current topics on cryofiltration technologies. Ther Apher. 2001 Aug;5(4):283-6.

27 Jansen M, Schmaldienst S, Banyai S, Quehenberger P, Pabinger I, Derfler K, et al. Treatment of coagulation inhibitors with extracorporeal immunoadsorption (Ig-Therasorb). $\mathrm{Br}$ J Haematol. 2001 Jan;112(1):91-7.

28 Leebmann J, Roeseler E, Julius U, Heigl F, Spitthoever R, Heutling D, et al.; Pro(a)LiFe Study Group*. Lipoprotein apheresis in patients with maximally tolerated lipid-lowering therapy, lipoprotein(a)-hyperlipoproteinemia, and progressive cardiovascular disease prospective observational multicenter study. Circulation. 2013 Dec;128(24):2567-76.

29 Stefanutti C, Vivenzio A, Di Giacomo S, Mazzarella B, Ferraro PM, Abbolito S. Treatment of symptomatic hyperLp(a)lipidemia with LDL-apheresis vs. usual care. Transfus Apheresis Sci. 2010 Feb;42(1):21-6.

30 Zenti MG, Stefanutti C. Effects of selective H.E.L.P. LDL-apheresis on plasma inflammatory markers concentration in severe dyslipidemia: implication for anti-inflammatory response. Cytokine. 2011 Dec;56(3):850-4.

31 Bosch T, Lennertz A, Schenzle D, Dräger J; Direct adsorption of lipoproteins (DALI) study group. Direct adsorption of low-density lipoprotein and lipoprotein(a) from whole blood: results of the first clinical long-term multicenter study using DALI apheresis. J Clin Apher. 2002;17(4):161-9.

32 Ritter MM, Parhofer KG. Extracorporal lowdensity lipoprotein elimination by immunoadsorption. Ther Apher Dial. 2003 Jun;7(3): 370-2.

33 Fernández-Mosteirín N, Salvador-Osuna C, García-Erce JA, Orna E, Pérez-Lungmus G, Giralt M. [Comparison between phlebotomy and erythrocytapheresis of iron overload in patients with HFE gene mutations]. Med Clin (Barc). 2006 Sep;127(11):409-12.

34 Rombout-Sestrienkova E, van Noord PA, van Deursen CT, Sybesma BJ, Nillesen-Meertens AE, Koek GH. Therapeutic erythrocytapheresis versus phlebotomy in the initial treatment of hereditary hemochromatosis - A pilot study. Transfus Apheresis Sci. 2007 Jun;36(3): 261-7.

35 Rombout-Sestrienkova E, Nieman FH, Essers BA, van Noord PA, Janssen MC, van Deursen $\mathrm{CT}$, et al. Erythrocytapheresis versus phlebotomy in the initial treatment of HFE hemochromatosis patients: results from a random- ized trial. Transfusion. 2012 Mar;52(3): 470-7.

36 Beth SH. Red cell exchange and other therapeutic alterations of red cell mass. In: McLeod $\mathrm{BC}$, et al., editors. Apheresis principles and practise. 3 ed. Bethesda: AABB; 2010.

37 Kalff A, Dowsing C, Grigg A. The impact of a regular erythrocytapheresis programme on the acute and chronic complications of sickle cell disease in adults. Br J Haematol. 2010 Jun; 149(5):768-74.

38 Ballas SK, Lyon D. Safety and efficacy of blood exchange transfusion for priapism complicating sickle cell disease. J Clin Apher. 2016 Feb; 31(1):5-10.

39 Auer-Hackenberg L, Staudinger T, Bojic A, Locker G, Leitner GC, Graninger W, et al. Automated red blood cell exchange as an adjunctive treatment for severe Plasmodium falciparum malaria at the Vienna General Hospital in Austria: a retrospective cohort study. Malar J. 2012 May;11(1):158.

40 Auer-Hackenberg L, Winkler S, Graninger W, Worel N, Ramharter M. Current evidence and future of automated erythrocyte exchange in the treatment of severe malaria. Wien Klin Wochenschr. 2012 Nov;124(S3 Suppl 3):23-6.

41 Santos B, Portugal R, Nogueira C, Loureiro M. Hyperhemolysis syndrome in patients with sickle cell anemia: report of three cases. Transfusion. 2015 Jun;55(6 Pt 2):1394-8.

42 Turner JM, Kaplan JB, Cohen HW, Billett $\mathrm{HH}$. Exchange versus simple transfusion for acute chest syndrome in sickle cell anemia adults. Transfusion. 2009 May;49(5): 863-8.

43 Melton CW, Haynes J Jr. Sickle acute lung injury: role of prevention and early aggressive intervention strategies on outcome [vii.]. Clin Chest Med. 2006 Sep;27(3):487-502.

44 Swerdlow PS. Red cell exchange in sickle cell disease. Hematology (Am Soc Hematol Educ Program). 2006;2006(1):48-53.

45 Yazdanbakhsh K, Ware RE, Noizat-Pirenne F. Red blood cell alloimmunization in sickle cell disease: pathophysiology, risk factors, and transfusion management. Blood. 2012 Jul; 120(3):528-37.

46 Worel N, Greinix HT, Supper V, Leitner G, Mitterbauer M, Rabitsch W, et al. Prophylactic red blood cell exchange for prevention of severe immune hemolysis in minor ABOmismatched allogeneic peripheral blood progenitor cell transplantation after reduced-intensity conditioning. Transfusion. 2007 Aug; 47(8):1494-502.

47 Petz LD. Immune hemolysis associated with transplantation. Semin Hematol. $2005 \mathrm{Jul}$; 42(3):145-55.

48 Ganzel C, Becker J, Mintz PD, Lazarus HM, Rowe JM. Hyperleukocytosis, leukostasis and leukapheresis: practice management. Blood Rev. 2012 May;26(3):117-22.

49 Blum W, Porcu P. Therapeutic apheresis in hyperleukocytosis and hyperviscosity syndrome. Semin Thromb Hemost. 2007 Jun; 33(4):350-4

50 Zuckerman T, Ganzel C, Tallman MS, Rowe JM. How I treat hematologic emergencies in adults with acute leukemia. Blood. 2012 Sep; 120(10):1993-2002.
51 Bug G, Anargyrou K, Tonn T, Bialleck H, Seifried E, Hoelzer D, et al. Impact of leukapheresis on early death rate in adult acute myeloid leukemia presenting with hyperleukocytosis. Transfusion. 2007 Oct;47(10):1843-50.

52 Porcu P, Danielson CF, Orazi A, Heerema NA, Gabig TG, McCarthy LJ. Therapeutic leukapheresis in hyperleucocytic leukaemias: lack of correlation between degree of cytoreduction and early mortality rate. Br J Haematol. 1997 Aug;98(2):433-6.

53 Saniabadi AR, Hanai H, Suzuki Y, Ohmori T, Sawada K, Yoshimura N, et al. Adacolumn for selective leukocytapheresis as a non-pharmacological treatment for patients with disorders of the immune system: an adjunct or an alternative to drug therapy? J Clin Apher. 2005 Oct;20(3):171-84

54 Waitz G, Petermann S, Liebe S, Emmrich J, Ramlow W. Reduction of dendritic cells by granulocyte and monocyte adsorption apheresis in patients with ulcerative colitis. Dig Dis Sci. 2008 Sep;53(9):2507-15.

55 Sacco R, Romano A, Mazzoni A, Bertini M, Federici G, Metrangolo S, et al. Granulocytapheresis in steroid-dependent and steroid-resistant patients with inflammatory bowel disease: a prospective observational study. J Crohn's Colitis. 2013 Dec; 7(12):e692-7.

56 Leitner GC, Vogelsang H. Pharmacologicaland non-pharmacological therapeutic approaches in inflammatory bowel disease in adults. World J Gastrointest Pharmacol Ther. 2016 Feb;7(1):5-20.

57 Namba K, Sonoda KH, Kitamei H, Shiratori K, Ariyama A, Iwabuchi K, et al. Granulocytapheresis in patients with refractory ocular Behcet's disease. J Clin Apher. 2006 Jul;21(2):121-8.

58 Worel N, Leitner G. Clinical Results of Extracorporeal Photopheresis. Transfus Med Hemother. 2012 Aug;39(4):254-62.

59 Wollina U, Lange D, Looks A. Short-time extracorporeal photochemotherapy in the treatment of drug-resistant autoimmune bullous diseases. Dermatology. 1999; 198(2): 140-4.

60 Knobler R, Jantschitsch C. Extracorporeal photochemoimmunotherapy in cutaneous $\mathrm{T}$ cell lymphoma. Transfus Apheresis Sci. 2003 Feb;28(1):81-9.

61 Abreu MT, von Tirpitz C, Hardi R, Kaatz M, Van Assche G, Rutgeerts P, et al.; Crohn's Disease Photopheresis Study Group. Extracorporeal photopheresis for the treatment of refractory Crohn's disease: results of an openlabel pilot study. Inflamm Bowel Dis. 2009 Jun;15(6):829-36.

62 Knobler R, Berlin G, Calzavara-Pinton P, Greinix H, Jaksch P, Laroche L, et al. Guidelines on the use of extracorporeal photopheresis. J Eur Acad Dermatol Venereol. 2014 Jan;28 Suppl 1:1-37.

63 Yakut E, Jakobs C, Peric A, Michel G, Baal N, Bein G, et al. Extracorporeal photopheresis promotes IL- $1 \beta$ production. J Immunol. 2015 Mar;194(6):2569-77.

64 Spisek R, Gasova Z, Bartunkova J. Maturation state of dendritic cells during the extracorporeal photopheresis and its relevance for the treatment of chronic graft-versus-host disease. Transfusion. 2006 Jan;46(1):55-65. 
65 Berger CL, Hanlon D, Kanada D, Girardi M, Edelson RL. Transimmunization, a novel approach for tumor immunotherapy. Transfus Apheresis Sci. 2002 Jun;26(3):20516.

66 Greinix HT, Worel N, Just U, Knobler R. Extracorporeal photopheresis in acute and chronic graft-versus-host disease. Transfus Apheresis Sci. 2014 Jun;50(3):349-57.

67 Greinix HT, Knobler RM, Worel N, Schneider B, Schneeberger A, Hoecker P, et al. The effect of intensified extracorporeal photochemotherapy on long-term survival in patients with severe acute graft-versus-host disease. Haematologica. 2006 Mar;91(3):405-8.

68 Barr ML, Meiser BM, Eisen HJ, Roberts RF, Livi U, Dall'Amico R, et al.; Photopheresis Transplantation Study Group. Photopheresis for the prevention of rejection in cardiac transplantation. N Engl J Med. 1998 Dec; 339(24):1744-51.
69 Chih S, Tinckam KJ, Ross HJ. A survey of current practice for antibody-mediated rejection in heart transplantation. Am J Transplant. 2013 Apr;13(4):1069-74.

70 Morrell MR, Despotis GJ, Lublin DM, Patterson GA, Trulock EP, Hachem RR. The efficacy of photopheresis for bronchiolitis obliterans syndrome after lung transplantation. J Heart Lung Transplant. 2010 Apr;29(4): 424-31.

71 Tefferi A. Polycythemia vera: a comprehensive review and clinical recommendations. Mayo Clin Proc. 2003 Feb;78(2):17494.

72 Marques MB, Singh N, Reddy VV. Out with the bad and in with the good; red cell exchange, white cell reduction, and platelet reduction. J Clin Apher. 2014 Aug;29(4): 220-7.
73 Barbui T, Barosi G, Grossi A, Gugliotta L, Liberato LN, Marchetti M, et al. Practice guidelines for the therapy of essential thrombocythemia. A statement from the Italian Society of Hematology, the Italian Society of Experimental Hematology and the Italian Group for Bone Marrow Transplantation. Haematologica. 2004 Feb;89(2):215-32.

74 Elliott MA, Tefferi A. Thrombosis and haemorrhage in polycythaemia vera and essential thrombocythaemia. Br J Haematol. 2005 Feb; 128(3):275-90.

75 Thiele J, Kvasnicka HM. The 2008 WHO diagnostic criteria for polycythemia vera, essential thrombocythemia, and primary myelofibrosis. Curr Hematol Malig Rep. 2009 Jan; 4(1):33-40.

76 Schafer AI. Thrombocytosis. N Engl J Med. 2004 Mar;350(12):1211-9.

77 Duvall D. Therapeutic cytapheresis: too many platelets, too many white blood cells. J Clin Apher. 2011;26(1):47-52. 